\title{
CULTURA ORGANIZACIONAL E INTELIGENCIA COMPETITIVA EN UNA INSTITUCIÓN DE EDUCACIÓN SUPERIOR DEL NORTE DE MÉXICO
}
Organizational culture and competitive intelligence in a higher education institution of northern Mexico

Armando Esquinca Morenoํㅜㄹ Carmen Patricia Jiménez Terrazas², Hugo Gaggiotti ${ }^{3}$

Fecha de recepción: 21 de febrero del 2018

Fecha de aceptación: 10 de abril del 2018

1-Nacionalidad: Mexicana. Grado: Maestría. Especialización: Administración. Adscripción: Universidad Autónoma de Chihuahua. Correo electrónico: a.esquinca@hotmail.com . (D) ORCID: http://orcid.org/0000-0002-8396-589X

2- Nacionalidad: Mexicana. Grado: Doctorado. Especialización: Administración. Adscripción: Universidad Autónoma de Ciudad Juárez. Correo electrónico: pjimenez@uacj.mx . (D) ORCID: http://orcid.org/0000-0001-8242-5421

3- Nacionalidad: Británica. Grado: Doctorado. Especialización: Administración. Adscripción: University of the West of England. Correo electrónico: hugo.gaggiotti@uwe.ac.uk. (D) ORCID: http://orcid.org/0000-0002-7892-1649 


\section{Resumen}

El objetivo es describir la cultura organizacional (CO) de directivos, administrativos y docentes de una IES en el norte de México y su visión de la inteligencia competitiva (IC) en el acopio de información para el logro de objetivos institucionales. Para lo cual se realizó un estudio cualitativo, de caso, con entrevistas semiestructuradas y teoría fundamentada, en una muestra teórica de 14 participantes y documentos institucionales. La codificación fue teórica selectiva con un modelo hipotético-deductivo basada en los tipos de CO de Cameron y Quinn y el ciclo de la IC. Se encontró que los resultados de CO de mercado que proyecta el plan institucional solo corresponden a lo que mencionan los administrativos y la IC en la proyección al 2021 como institucionalizada y normada, aunque actualmente falta conocimiento sobre la IC de la institución y unificación de criterios sobre quién y que estrategias realizan. El documento incluye recomendaciones para el cambio cultural que se requiere en la proyección 2021.

Palabras clave: cultura organizacional, inteligencia competitiva, instituciones de educación superior.

\section{Abstract}

The objective is to describe the organizational culture (OC) of managers, administrators and teachers of higher institution in the north of Mexico and their vision of competitive intelligence (CI) in the collection of information for the achievement of institutional objectives. A qualitative and case study was conducted, with semi-structured interviews and grounded theory, in a theoretical sample of 14 participants and institutional documents. The coding was selective theoretical with a hypothetico-deductive model based on Cameron and Quinn's OC types and the CI cycle. It was found that the results of market OC that the institutional plan projects, only correspond to what the administrative mention and CI in the 2021 projection as institutionalized and regulated, although currently lack of knowledge about the CI of the institution and unification of criteria on who and what strategies they carry out. The document includes recommendations for the cultural change that is required in the 2021 projection.

Keywords: organizational culture, competitive intelligence, higher education institutions. 


\section{Introducción}

unque la cultura organizacional $(\mathrm{CO})$ ha sido estudiada desde antes de mediados del siglo
XX por áreas de la ciencia no relacionadas a la administración, fue hasta la década de los
sesentas cuando se presentan de forma clara los primeros enfoques organizacionales. Este concepto fue evolucionando con el paso del tiempo identificando cinco etapas: la primera incorpora cuestiones sociológicas estudiando por ejemplo, como se convivía en la organización, pues a través de la CO se manifestaba la forma en que la misma organización le daba sentido o significado; en la segunda (a partir de 1983 a 1985) se observa a la CO como un objetivo colectivo de las organizaciones; en la tercera (de 1985 a 1991) como un elemento estratégico y la influencia que tienen desde la gerencia; en la cuarta (1991 a 2010) se conceptualiza a la CO como una identidad colectiva con significados compartidos, como un activo inteligente que ayuda a enfrentar incertidumbres derivadas de factores tanto externos como internos.

Este artículo se centra en la quinta etapa del concepto de CO que se sitúa en el tiempo a partir de los años 2010 como predictor que orienta, gestiona y controla la organización, además de que permite predecir la dirección de la misma. En ese sentido, la CO incorpora o se relaciona con herramientas, métodos o procedimientos que favorezcan su gestión como los sistemas de información, gestión del conocimiento o la inteligencia competitiva.

El estudio de la CO en las Instituciones de Educación Superior (IES), se ha desarrollado a lo largo de cada una de las etapas anteriormente mencionadas y desde los enfoques cualitativos y cuantitativos. Bikmorardi, Brommels, Shoghli, Zavarech y Masiello (2009: 417) definen la cultura académica como un grupo de actitudes, creencias y valores que le dan integridad a un grupo específico de académicos, lo que genera subculturas dentro de una misma CO de las IES. Dill (2012: 223) identifica tres niveles de cultura en la educación superior: cultura de la disciplina, cultura de la empresa (o cultura organizacional) y cultura de la profesión académica y/o sistema nacional.

Desde un enfoque de cultura de la empresa (o CO) debido a la influencia de factores externos a las IES a nivel internacional y en México no ha sido la excepción, como el énfasis por la calidad universitaria desde los años ochenta, traducido en evaluaciones y acreditaciones con indicadores de calidad y competitividad, ha generado un "traslape" de las visiones y funciones de los actores principales que las operacionalizan: los administrativos y docentes, ya que mientras unos buscan la eficiencia y gestión misma de la organización, los otros la ven a través de la transmisión de conocimientos y el avance científico.

Este "traslape" debido a sus diferentes objetivos, enfoques o visiones de una misma entidad (la IES) crean un efecto de coalición debido a esas diferentes funciones que desarrollan. Debido a esto la pregunta de investigación que guía este estudio es ¿Cómo conciben la cultura organizacional y la inteligencia competitiva los miembros de una organización, en particular directivos, administrativos y docentes en una IES pública en el norte de México? 


\section{Revisión de literatura}

Los estudios sobre cultura organizacional e inteligencia competitiva se remontan a finales de los años noventa del siglo pasado y hacen referencia a la competencia global a la que se han visto sometidas organizaciones, empresas e instituciones, ya sea en el sector privado o público, así como a la rapidez de los cambios tecnológicos que incorporan como un punto de quiebre que las hallevado a modificar su forma de hacer el trabajo, incorporando la inteligencia competitiva como una de ellas y entendiéndola como "la recopilación sistemática, análisis, almacenamiento, difusión y protección de información específicamente adaptada tanto para operativos como gerentes estratégicos" (Cory, 1996: 45).

En ese sentido, este creciente interés en la CO e IC de acuerdo a Simon (1999:67), se justifica debido a que los nuevos procesos que adopten con nueva o diferente tecnología, puedan ser una barrera o verse favorecidos por la cultura que se desarrolle, por lo que puede asumirse que algunas $\mathrm{CO}$ son más propicias para realizar una efectiva IC mientras que otras, son menos propias para un alto desarrollo de la IC. Soto (2001:31) en ese mismo sentido, pero ya considerando el uso de sistemas en una organización, considera en gran medida la CO como "pegamento" que une las organizaciones y controla sus operaciones, reemplazando de alguna manera las políticas y procedimientos establecidos, ya que influye en que las cosas se hagan de manera eficiente y efectiva hasta en la forma en que se realicen los análisis de IC.

Kbrom (2004: II) también menciona que el nivel de desarrollo formal o informal de la inteligencia competitiva es influenciado por la cultura organizacional en dos sentidos: primero por la relación entre las dimensiones de la CO (involucramiento del empleado, recursos humanos, enfoque organizacional, canales de comunicación, recompensa y confianza) e IC; segundo, en términos de los puntajes de estas relaciones. Moffat y Fleisher (2003: 275-278) por otro lado, identifican características culturales corporativas negativas y positivas de IC.

Otros autores han enfocado el estudio de CO e IC desde la ética. Giuostozzi y Martens (2011: 1) examinan la importancia de un marco de referencia ético relacionado a la CO para el uso de softwares $\mathrm{y}$ actividades en internet de IC, considerando las implicaciones legales y publicas del uso de la información. En ese sentido, la Sociedad de Inteligencia Competitiva la define como un método de recolección de información ética y moral (Petrisor y Strain, 2013: 100).

Enfoques más recientes del estudio de $\mathrm{CO}$ e IC se relacionan con la manera en que la IC debe incorporarse a la CO. Petrisor y Strain (2013: 102-103) consideran que, para introducir nuevas iniciativas de trabajo como la IC, es importante "ajustarlas" a la CO macro de la organización, en lugar de imponerlas, lo que permite que algunas personas vayan aprendiendo sobre la IC y conduzcan este aprendizaje a los demás participantes. Wang y Borges (2013: 80) en relación a los profesionales que trabajan la IC, mencionan la importancia de que conozcan y entiendan los símbolos, ritos y mitos (elementos de la CO) de sus clientes o compañeros de trabajo, que les permita identificar sus necesidades de información.

Otros autores sobre CO e IC hacen énfasis en la importancia de distinguir los diferentes tipos de cultura al momento de introducir la IC en una empresa. En ese sentido, Chebbi y Ammar (2015; 37-38) proponen un modelo conceptual al que llaman "modelo innovador de CO e IC" utilizando el marco de valores en competencia de Cameron y Quinn (2006: 31), para catalogar, identificar y diferenciar las diferentes culturas y su relación con las diferentes etapas del proceso de IC. Llevando este modelo conceptual a la práctica, Bogdanowicz (2014: 53-58) realiza un estudio basado en el marco de valores en competen- 
cia de Cameron y Quinn y su alineación con las metas estratégicas de las empresas, donde menciona que uno de los elementos más importantes en un proceso de cambio es realizar un diagnóstico correcto de las diferencias entre las características de la cultura actual y la que desea modificarse con esos cambios. También Cekuls (2015: 248) basado en la teoría de CO de Cameron y Quinn (2006), el proceso de IC de Calof (1999) y Breakspeare y a la transformación cultural de Barret, estudia el rol de los valores de la gerencia, los cambios en la cultura organizacional en la implementación de la IC, encontrando que la manera exitosa consiste en involucramiento del personal en la toma de decisiones, soporte técnico, comunicación de la visión y crear una atmosfera amigable.

Finalmente, en relación a los estudios de CO e IC es importante señalar que, aunque la mayoría de los anteriormente mencionados son artículos teóricos o ensayos, de los empíricos ninguno se llevó a cabo en México o en IES: en industrias de manufactura en Eritrea (Kbrom, 2004: 38); compañía de telecomunicaciones en Polonia (Bogdanowicz, 2014: 53) y compañías de Letonia (Cekuls, 2015: 244), por lo que el presente estudio es pertinente a realizar en un contexto y población escasamente estudiado, como lo son las Instituciones de Educación Superior en México

\subsection{Cultura organizacional}

La definición de cultura organizacional la conforman elementos como los valores, hábitos, ritos, héroes, símbolos, ritos, reglas, normas y políticas para el logro de objetivos, metas, resultados y tareas organizacionales; vistos como elementos estratégicos, con identidad colectiva y significados compartidos, influenciados por la gerencia y como predictor que orienta, gestiona y controla (poder) la organización.

En estos elementos de la definición de la CO, Denison y Spreitzer (1991: 5), señalan que en el centro de la organización se encuentran los valores y las creencias están en la raíz de los sistemas de la organización. Desde la interpretación del constructivismo social Hatch y Schultz (1997: 357), señalan que los valores reglas, normas y rituales, no solo se pueden ver ontológicamente, sino cómo influyen en la imagen e identidad de las organizaciones, concretamente en la manera que perciben y piensan acerca de su organización. Es un colectivo de entendimiento de valores y características. En ese contexto en las organizaciones presentan diferentes niveles de subjetividad donde presentan un proceso, de "como" le dan sentido o "sensemeking", que se da conjuntamente, es decir: organización y la manera de que las personas las perciben están en un mismo plano (Weick, 1995: 71).

En relación a los cambios de la organización, Schein (2004: 225) menciona que se realizan a través del líder, quien impone valores y creencias en el grupo y los define para otras generaciones como aceptables para los miembros. Aunque Alvensson, (2002: 171) considera que el cambio en la cultura es espontaneo y una constante revalorización que no necesariamente es iniciada desde la administración o gerencia, sino de los diferentes miembros de grupos, que reaccionan ante nuevos cambios. Denison y Spreitzer (1991: 5) también mencionan que un agente de cambio o un cambio en la organización, necesariamente debe identificar como es la relación de los valores subrepticios, que conectan a los miembros de la organización, y su impacto en el trabajo de equipo.

Varios teóricos y autores de la CO han desarrollado y diseñado marcos conceptuales para definir sus diferentes tipos o tipologías, modelos o herramientas de medición. Para propósitos de estudio del presente 
artículo, nos centraremos en el modelo teórico de Cameron y Quinn (2006: 31) denominado marco de valores en competencia basado en los valores que los individuos tienen dentro de una organización relacionados a su desarrollo y a la manera en como las cosas funcionan dentro de la misma, de tal forma que la entienden, dimensionan y clasifican en cuatro tipos de cultura: de clan, mercado, adhocratica y jerárquica. Estos diferentes tipos de cultura se basan o esquematizan en dos ejes que forman cuatro cuadrantes (ver figura 1). El eje vertical representa que tal flexible o centralizado es el control de la organización. Las empresas muy centralizadas tienen, por ejemplo, sus procesos y procedimientos documentados para llevarlos a cabo de esa manera. El eje horizontal describe si el enfoque de la organización es interno o externo. Las empresas que se enfocan de manera externa tienden a regirse por las fuerzas del mercado, nuevos clientes o competidores.

\section{Figura 1. Marco de valores en competencia}

\section{Flexibilidad y discresión}

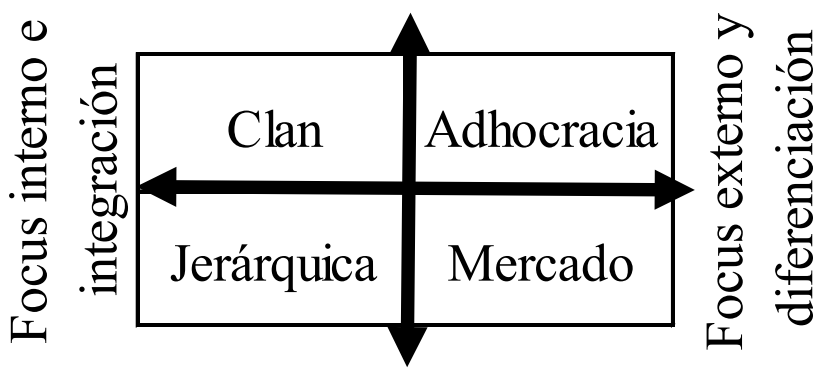

\section{Estabilidad y control}

Fuente: Cameron y Quinn (2006)

Estos cuatro tipos de cultura de Cameron y Quinn (2006: 94) se distinguen por:

1. Cultura de Clan: en donde se desarrolla un "ambiente familiar" y se comparten la toma de decisiones, existe orden y control versus flexibilidad y discreción enfocada en lo interno, los líderes en esta dimensión cultural son facilitadores y creadores de trabajo en equipo.

2. Cultura de mercado: esta expresión va enfocada hacia búsqueda de resultados, objetivos, metas, una orientación de competitividad, en la lógica de estabilidad y control enfocado a lo externo, este ejercicio de liderazgo es de forma autoritaria, los líderes se entienden como conductores duros, con una actuación que presiona hacia resultados.

3. Cultura de Adhocracia: una dimensión hacia la flexibilidad y discreción con enfoque externo, el liderazgo es orientado hacia la creatividad, la innovación y la visión, los valores del líder son la innovación en la investigación y la efectividad.

4. Cultura jerárquica: las dimensiones son la estabilidad y control con enfoque interno, el liderazgo es ejercido con orientación controladora, de coordinación, monitoreo organizado, los valores conductores son la eficiencia en tiempos, constancia y uniformidad, para lograr la efectividad se basan en procesos establecidos. 
En relación a estos tipos de cultura, los autores mencionan que no existe una cultura buena o fuerte, pues esta clasificación no agota la realidad, pero si existen culturas dominantes, que se presentan constantemente en las instituciones, que pueden favorecer o entorpecer el desempeño de las mismas, cuando se enfrentan a cambios o amenazas

De acuerdo a este marco de valores en competencia, Cameron y Quinn (2006: 25) desarrollaron una herramienta que les permitiera perfilar las características dominantes de la CO de una organización mediante el instrumento de evaluación de cultura organizacional, donde cada tipo de cultura se define por seis dimensiones (ver tabla 1): 1.-las características dominantes, 2.- el liderazgo que se ejerce, 3.- administración de los empleados, 4.-la unión de la organización que le da cohesión, basados en la lealtad y el desarrollo de la misma, 5.-énfasis estratégico y 6.- como se obtiene el éxito de la misma

\begin{tabular}{|c|c|c|c|c|}
\hline & Clan & Adhocracia & Mercado & Jerárquica \\
\hline $\begin{array}{l}\text { Característica } \\
\text { dominante }\end{array}$ & $\begin{array}{c}\text { organizada como } \\
\text { familia }\end{array}$ & $\begin{array}{l}\text { dinámica, toma ries- } \\
\text { gos y emprendedora }\end{array}$ & $\begin{array}{c}\text { orientada al producto } \\
\text { y empleados }\end{array}$ & $\begin{array}{l}\text { formalizada, estructu- } \\
\text { rada, procedimientos }\end{array}$ \\
\hline Liderazgo & $\begin{array}{l}\text { mentores, facilitado- } \\
\text { res o figura paterna }\end{array}$ & $\begin{array}{c}\text { emprendedores, inno- } \\
\text { vadores o tomadores } \\
\text { de riesgos }\end{array}$ & $\begin{array}{c}\text { productores, duros y } \\
\text { competidores }\end{array}$ & $\begin{array}{l}\text { coordinadores, orga- } \\
\text { nizadores y eficientes }\end{array}$ \\
\hline $\begin{array}{l}\text { Administración } \\
\text { de empleados }\end{array}$ & $\begin{array}{l}\text { equipo, consenso y } \\
\text { participación }\end{array}$ & $\begin{array}{l}\text { riesgos individuales, } \\
\text { innovadores, flexibili- } \\
\text { dad, únicos }\end{array}$ & $\begin{array}{l}\text { competitividad, } \\
\text { manejo difícil, metas, } \\
\text { dirección y logros }\end{array}$ & $\begin{array}{l}\text { control cuidadoso del } \\
\text { desempeño, posición } \\
\text { y predictibilidad }\end{array}$ \\
\hline Cohesión & $\begin{array}{l}\text { lealtad y mutua } \\
\text { confianza }\end{array}$ & $\begin{array}{c}\text { orientados a la inno- } \\
\text { vación y desarrollo }\end{array}$ & $\begin{array}{c}\text { énfasis en producción } \\
\text { y acompañamiento } \\
\text { de metas }\end{array}$ & $\begin{array}{l}\text { reglas formales y } \\
\text { políticas }\end{array}$ \\
\hline $\begin{array}{l}\text { Énfasis estraté- } \\
\text { gico }\end{array}$ & $\begin{array}{l}\text { desarrollo humano, } \\
\text { alta confianza, aper- } \\
\text { tura y participación }\end{array}$ & $\begin{array}{l}\text { adquisición de nuevos } \\
\text { recursos y nuevos } \\
\text { retos }\end{array}$ & $\begin{array}{c}\text { acciones competiti- } \\
\text { vas y logro }\end{array}$ & $\begin{array}{l}\text { permanencia y estabi- } \\
\text { lidad }\end{array}$ \\
\hline Criterios de éxito & $\begin{array}{l}\text { desarrollo de } \mathrm{RH} \text {, } \\
\text { trabajo en equipo y } \\
\text { enfoque en la gente }\end{array}$ & $\begin{array}{c}\text { productos únicos y } \\
\text { nuevos }\end{array}$ & $\begin{array}{c}\text { penetración y cuota } \\
\text { de mercado }\end{array}$ & $\begin{array}{c}\text { eficiencia (bajos } \\
\text { costos, programación } \\
\text { constante) }\end{array}$ \\
\hline
\end{tabular}

Fuente: Cameron y Quinn (2006)

\subsection{Inteligencia competitiva}

La Inteligencia Competitiva se define como un método (Petrisor y Strain, 2013: 100) o proceso (Wang y Borges, 2013: 76) o programa sistemático y ético para recolectar, analizar y administrar cualquier combinación de datos, información y conocimiento relacionado al ambiente de negocios en el que opere la organización, con la cuál le otorgue una ventaja competitiva o favorezca la toma de decisiones. Su principal función es generarle a las empresas una alerta estratégica temprana (Prior, 2018). 
La obtención de los recursos de información de acuerdo al ciclo de García (2011: 98), se concreta en los siguientes puntos:

1. Detección y recogida de información

2. Organización y almacenamiento de la información centrada en las fuentes y los canales para la adquisición de la información

3. Interpretación y análisis

4. Comportamiento adaptativo e inteligencia general

En la fase de detención de las necesidades, son relevantes los procedimientos para recoger los temas sobre los cuales se busca la información e ir actualizando el inventario de necesidades. En sí, la dirección de una organización está determinada por entender las necesidades de la misma, que le permita generar una estrategia.

De acuerdo con García (2011: 71), en la fase de recogida de información, los temas de interés son:

a) Definición de los procedimientos para localizar, actualizar y/o validar las fuentes empleadas;

b) Tipo de información de fuentes utilizadas, incluidas las redes sociales, criterios de selección y su relevancia para las necesidades detectadas;

c) Los canales de detención de la información empelada formales o informales, estos influyen en la orientación de la función proactiva o reactiva, táctica o estratégica.

En la fase de organización y almacenamiento de la información, los temas de interés son:

a) Los procedimientos integrados de gestión de la información existentes en todos los departamentos y unidades de gestión;

b) Las tecnologías de soporte disponibles en la organización.

En la fase de interpretación y análisis de la información:

a) Los productos y servicios de información, con valor añadido, elaborados u obtenidos disponibles en la organización;

b) Los canales de difusión de estos productos y servicios;

c) Los espacios y las estructuras dentro de la organización disponible para compartir, interpretar y analizar información.

d) Y de las técnicas de análisis para extraer inteligencia.

En relación a las diferentes culturas de inteligencia competitiva Moffat y Fleisher (2003: 275-278) identifican características culturales corporativas negativas de IC independientemente de que la IC se practique de manera informal en alguna empresa o no cuente con personas o departamentos cuya responsabilidad sean las actividades de IC, siendo éstas: 
1. No reconocen su propia mentalidad IC o manejan una mentalidad inapropiada

2. Sufren de la llamada "enfermedad net" que se refiere a cuando las empresas piensan que la IC empieza y termina con el internet

3. Practican deliberadamente o permiten prácticas poco éticas de IC como tergiversar la identidad de alguien o engañar para obtener información confidencial, ofrecer sobornos, compensaciones o favores para intercambiar información secreta, hackeo de computadoras o intervención de líneas telefónicas

4. Implementan "silo" IC cuando se niegan a reconocer los beneficios de usar la IC en toda la organización apoyándose en redes coordinadas y descentralizadas

5. Desarrollan análisis de IC pobres o de manera apresurada

6. Fomentan el desarrollo de al menos cuatro tipos de barreras de IC: recursos, estructura y procesos, entendimiento de la IC y de actitudes. En las barreras de recursos frecuentemente es por fondos o personal insuficiente o con pocas habilidades en el área de IC. Las barreras de estructura y proceso hacen referencia a estructuras inapropiadas (o inexistentes) dentro de la organización o mecanismos - procesos incompletos o inapropiados. La barrera del entendimiento hace referencia al limitado conocimiento de los propósitos y significados del IC entre el personal de la organización. Y las barreras de actitudes se refieren a que las personas no confían en la IC y se les imposibilita compartir información (Calof, 1999:71).

En cuanto a las organizaciones IC positivas se encontró las siguientes características:

1. Se realizan auditorias en las cuales se evalúa la IC total de la organización, para realizar los objetivos de su estrategia competitiva.

2. Se llevan a cabo un balance real de los resultados, estableciendo objetivos tangibles y medibles, relacionados con la misión y la visión y estrategia de la organización.

3. Realizan un mapeo basados en estándares de calidad.

4. Benchmarketing. Se llevan a cabo estrategias y prácticas de IC y comparan resultados con otras organizaciones.

En Culturas positivas de IC, los encargados de tomar decisiones de las diferentes áreas en las organizaciones, regularmente buscan medir, el desempeño de la misma, tales como:

La cantidad de información, la calidad de la misma, el costo-beneficio de la utilización de la IC y el tiempo que le toma analizar (Moffact y Fleisher, 2003: 273-274).

\subsection{Información que les requieren a las IES para su calidad}

En México los organismos evaluadores y acreditadores especializados por áreas disciplinares y de calidad en investigación y la producción científica a nivel pregrado es Consejo para la Acreditación de la Educación Superior (COPAES) y a nivel posgrado el Programa Nacional de Posgrados de Calidad (PNPC), ambos avalados por la Secretaría de Educación Pública (SEP). 
El COPAES constituye el único organismo avalado por la Secretaria de Educación Pública (SEP) para otorgar la acreditación de los programas. El compromiso de COPAES es desarrollar el Sistema Nacional de Evaluación, Acreditación y certificación (COPAES, 2013), apoyado por los Comités Interinstitucionales para la Evaluación de la Educación Superior (CIEES), los cuales nacen en1991, sus actividades se enfocan en el diagnóstico y la evaluación de los programas educativos con base en la asignación de dos niveles: 1 y 2 . El nivel 1 es un programa de buena calidad y nivel 2 es un programa con deficiencias en aspectos fundamentales en su operación (CIEES, 2017).

El Programa Nacional de Posgrados de Calidad (PNPC) es un esfuerzo conjunto entre la Subsecretaría de Educación Superior de la Secretaría de Educación Pública y el Consejo Nacional de Ciencia y Tecnología, que acumula la experiencia adquirida en la evaluación del posgrado en México desde la década de los noventa y toma en cuenta las buenas prácticas internacionales en la materia. La Dirección de Posgrado del Consejo Nacional de Ciencia y Tecnología (CONACYT) ha diseñado, de manera participativa con grupos de enfoque las distintas modalidades que conforma la metodología de evaluación y seguimiento del PNPC (CONACYT, 2017).

La información que ambos organismos requieren a las IES en México, para este estudio se han agrupado en cuatro grandes rubros:

\section{1.- Producción científica/investigación/ patentes.}

Publicaciones científicas en razón de excelencia e impacto a los programas académicos, número de profesores PTC en el sistema nacional de investigadores (PNPC-CONACYT), El número de patentes registradas. La producción que se evalúa es en base en la calidad de esta producción que a la vez indica un alto grado de investigación.

\section{2.- Acreditación/ evaluación}

La acreditación busca encontrar áreas de oportunidad para lograr la mejora continua con base en tres ejes: modernización innovación y vinculación (CIEES, PNPC).

\section{3.- Recursos/Infraestructura}

El personal académico altamente valorado por su desempeño y perfil deseable es el recurso principal, que permite obtener a su vez redes de vinculación que la institución busca, desarrollar la infraestructura operacional, como laboratorios, aulas y auditorios espacios adecuados refleja el grado de compromiso de la institución para el desarrollo de la calidad educativa. Algunos de los métricos de los diferentes acreditadores/evaluadores son: fundamentos y condiciones de operación, infraestructura y servicios (CIEES), Número de Profesores de Tiempo Completo, Número de PTC con perfil deseable, numero de cuerpos académicos del Núcleo Académico Base (NAB), vinculación de la IES como movilidad, intercambio, estancias académicas (COPAES).

\section{4.- Colaboración/internacionalización}

Las redes académicas temáticas, la investigación científica, exige un grado de colaboración nacional e internacional, la colaboración con diferentes órganos del Estado y empleadores del sector productivo, permite a las IES un mayor acercamiento con la sociedad misma que se ve reflejado con un currículo cada vez más flexible-específico para las necesidades regionales, nacionales e internacionales. Algunos de los métricos de los diferentes acreditadores/evaluadores son: Publicaciones con profesores de universidades extranjeras, revistas científicas, colaboración internacional, considera la clasificación del Programa nacional de programas de calidad, conforme a competencias internacionales, son desarrollo, consolidado 
y recién creado. Académicos de las instituciones en el (SNI) Sistema Nacional de Investigadores (PNPC), Académicos con alta producción académica en archivos ricos y visibles, Investigación que se refleja en revistas científicas, vinculación e innovación (COPAES), currículo especifico, actividades de los estudiantes, así con vinculación con empleadores para prácticas profesionales y con las diferentes estancias de gobierno para la realización del servicio social (CIEES).

\section{Métodos}

El objetivo del presente artículo es adentrarse al debate teórico-conceptual en torno a la Cultura Organizacional y la Inteligencia competitiva, con la intención de aportar al tratamiento conceptual desde la descripción y la concepción de cultura organizacional de directivos, administrativos y docentes de una IES en el norte de México, así como su visión y enfoque del uso de la inteligencia competitiva como método para el acopio de información administrativa para el logro de objetivos académicos institucionales. Su abordaje metodológico es de corte cualitativo, en un estudio de caso, utilizando la teoría fundamentada, a través de entrevistas semi-estructuradas y documentos institucionales en una población de docentes, administrativos y directivos de una IES en el norte de México con una muestra teórica de 14 participantes.

La investigación es cualitativa, ya que como se plantea en el objetivo del presente estudio, busca la descripción y concepción de la CO e IC en una IES y este tipo de investigación privilegia la profundidad sobre la extensión ya que busca captar los sutiles matices de las experiencias vitales de los participantes (Vasilachis, 2006: 27). Es importante mencionar que aunque las clasificaciones de CO de Cameron y Quinn ya cuentan con un cuestionario cuantitativo y muchas de las investigaciones que utilizan esta clasificación prefieren hacerlo cuantitativamente, resulta pertinente buscar particularidades desde un abordaje y estrategia metodológica cualitativa, ya que a través de la aplicación y análisis de entrevistas se loga describir las diferentes percepciones de los rasgos de la clasificación con una determinación profunda de las características culturales de la organización.

El estudio de caso, es un tipo de investigación apropiado en situaciones en las que se desea estudiar intensivamente características básicas, la situación actual e interacciones con el medio de una o unas pocas unidades tales como individuos, grupos, instituciones o comunidades (Tamayo, 2002: 51). Han sido estudiados como casos instituciones como universidades, fabricas, hospitales, institutos de corrección, departamentos de sanidad, hermandades y grupos de negocios. En cada caso de atención se dirige a lo típico de ellos, destacando los factores que caracterizan al tipo. (Best, 1970: 101).

Un estudio de la teoría fundamentada se distingue por ser un estudio de un análisis abstracto del fenómeno, relacionado con una situación en particular (Creswell, 1998: 55). En el estudio el investigador propone una explicación general del fenómeno u ofrece una teoría, de la interacción y proceso contextualizado en la situación particular (Hernández, Fernández, \& Baptista, 2014: 472). Se entiende que la posición teórica de una investigación que se centra desde el posicionamiento de la teoría fundamentada se centra en un interaccionismo simbólico, en donde el origen del significado es un producto social, que se desarrolla a partir de las actividades de los individuos al convivir o interactuar en un espacio físico (Hernández, 2014: 189). 
Desde la teoría fundamentada, lo que el investigador cuestiona que es lo que sucede dentro de una organización, es una interpretación analítica desde el mundo de los participantes evaluándola desde cuatro criterios: 1) ajuste, esto es que encaje en la experiencia de los participantes, 2) funcionamiento, es decir que explique la mayor variedad posible, 3) relevancia al fenómeno en estudio, 4) la posibilidad de modificarse la propia teoría; que significa que esta teoría se pueda acomodar a nuevos hallazgos (de la Cuesta, 2006: 137).

La población es docentes, administrativos y directivos de una IES del norte de México, específicamente en el estado de Chihuahua del subsistema de Universidad Pública Estatal (UPE) que desarrolle funciones de docencia, generación y aplicación del conocimiento, extensión y difusión de la cultura, ya que pueden hacerles frente a indicadores de calidad y competitividad que les requieren información especificada en este estudio. En 1954 el Congreso del Estado expidió el decreto de su fundación (UACH, 2018a), cuenta con 15 facultades distribuidas geográficamente en 11 municipios del estado de Chihuahua (UACH, 2018b); a la fecha es evaluada por los organismos afiliados a COPAES y sus posgrados por PNPC.

La muestra cualitativa es teórica o teorética. De acuerdo a Cáceres (2003: 63) para la determinación de la muestra son importantes las siguientes reglas: determinar las unidades de análisis y retroalimentar las partes. Por el muestreo teórico el investigador selecciona casos a estudiar según su potencial para ayudar a refinar o expandir los conceptos o teorías ya desarrollados (Vasilachis, 2006: 84). Para acercarse al objeto de estudio planteado, en la muestra teórica la búsqueda de entrevistados, está relacionado al criterio del investigador de quien proporcione información relativa a la aproximación y construcción desarrollada en la teoría, donde se pueda determinar los atributos centrales de una organización (Cameron y Quinn, 2006: 63). Los criterios de inclusión y exclusión de acuerdo a Martín-Crespo y Salamanca (2007: 3) incluyen: 1.- Tiempo, actividades y actitudes del trabajo de campo de acuerdo a la accesibilidad de los investigadores y de los entrevistados, 2.- La representación adecuada de las personas a participar, ya que ningún medio es socialmente homogéneo y 3.- Definición de individuos relevantes de acuerdo al lugar y contexto. En ese sentido, se buscó entrevistar a docentes, directivos y administrativos (siendo estos administradores y coordinadores de área) directamente relacionados con el proceso de acreditación de los programas de estudio, además de los que están directamente relacionados con las estrategias de mejoramiento académico, académicos de tiempo completo, investigadores con un perfil deseable.

Una vez definida la unidad de análisis, el número aceptable de una muestra de acuerdo a los objetivos de la investigación, basándose en la proximidad y la profundidad de la respuesta, es de siete a diez participantes, que se pueden dividir en subgrupos determinados por la coherencia del grupo (Mejía, 2000: 176). Considerando ese parámetro, el tamaño de la muestra en la presente investigación es de 14 entrevistados: 4 directivos, 4 administrativos y 6 docentes.

Las técnicas de recolección de información son entrevistas semi-estructuradas y análisis de documentos estratégicos de la IES. De acuerdo con Blaxter, Hughes, Tight (2008: 208) la principal ventaja de la entrevista semiestructurada frente a la entrevista individual es la interacción entre los participantes que puede convertirse en limitación, si los participantes se sienten coartados por la presencia del grupo o por la dominancia de algunos miembros. También Bryman y Bell (2015: 484) plantean que, en este tipo de entrevistas, el entrevistador la realiza de acuerdo con tópicos que se deben de cubrir y siguiendo una serie de preguntas en la forma de una guía, pero con la posibilidad de que sea flexible. El análisis de documentos públicos de la IES provee al investigador de antecedentes que le permiten describir la institución 
y entender las decisiones estratégicas y administrativas de la misma, así como situarse en una línea del tiempo (Bryman y Bell, 2015: 561).

El documento estratégico de la IES a analizar, es el Plan de Desarrollo Universitario 2011-2021 (UACH, 2011) por ser el marco orientador de acciones y toma de decisiones de la institución y que además contiene la visión para el 2021 y un conjunto de políticas y estrategias para lograrlo, por lo que es un referente adecuado para el análisis de la CO e IC de esta investigación. En su misión se declaran como una organización socialmente responsable que coadyuvan en el desarrollo integral social y económico en aras de mejorar la calidad de vida, logrando la formación de personas integras, con valores universales y capaces de dar respuestas pertinentes y creativas. Mediante una planta académica de alto nivel y programas de reconocida calidad. Los valores y principios que describen son: una gestión activa y coherente entre el discurso institucional y la acción, que sea responsable en la generación y aplicación del conocimiento y aseguramiento de calidad. En la declaración de valores señalan: igualdad de oportunidades educativas relevantes y de buena calidad. La ética, la honestidad, verdad, tolerancia.

Sus estrategias descritas en sus doce ejes rectores son: 1.-aseguramiento de la calidad educativa, 2.mejora del nivel de perfil y nivel de habilidades de la planta académica 3.-Fortalecimiento de las capacidades para la generación y aplicación del conocimiento, 4.- Fomento a la colaboración e intercambio académico entre campus y dependencias de educación superior de la universidad y con otras IES, 5.Impulso a la internacionalización de las funciones universitarias, 6.- Promoción para el desarrollo de la universidad saludable, 7.- Fortalecimiento de los esquemas de vinculación y extensión universitaria, 8.- Fortalecimiento de la difusión de la cultura con un fuerte sentido social, 9.- Impulso de la participación social, 10.- Consolidación de una universidad incluyente, con valores socialmente responsables, comprometida con el cuidado y conservación del medio ambiente y con un fuerte sentido de identidad, 11.- Promoción del deporte, la actividad física, y la recreación y .- 12.- Fortalecimiento de la gestión. La descripción de la misión, valores y estrategias de esta IES permite para esta investigación conocer su identidad y miras institucionales con las cuales se describe al público en general.

En cuanto a la recolección de datos, las entrevistas se realizaron en el periodo agosto-diciembre 2017 con una duración de 50 a 110 minutos mediante el siguiente protocolo: primero contactando de manera personal a los entrevistados para definir fecha y hora de la entrevista, así como describirle brevemente el objetivo del estudio. Una vez que aceptaron participar y previo al inicio de la entrevista, se pidió su consentimiento informado por escrito, así como su autorización para grabar. La entrevista se siguió con el instrumento previamente elaborado de una guía que contenía una serie de preguntas con fundamentación teórica sobre $\mathrm{CO}$ e IC, además de un glosario de conceptos. Una vez concluida la entrevista, se procedió a la transcripción de la misma.

\section{Resultados}

La confiabilidad en un estudio cualitativo, debe reunir características diferentes a la metodología cuantitativa, pues se considera como una valorización interna del proceso. La forma en que se ha procesado la información y la valorización de los resultados es una de las formas de confiabilidad cualitativa. De acuerdo al proceso del manejo de la información, Martínez (2018: 5), considera describir con claridad la 
utilización de todos los medios técnicos para la realización de la investigación. En el presente estudio, una vez que el entrevistado firmó el consentimiento informado y dio su autorización para grabar la entrevista, se grabó en audio usando una Sony IC recorder y se transcribió la entrevista en su totalidad, apoyándose en la aplicación Dragon Naturally Speaking.

Una vez con la transcripción de todas las entrevistas, se utilizó el análisis del discurso, que es la técnica y reducción de datos que beneficia el enfoque cualitativo propio de la teoría fundamentada (Caceres, 2003: 57). Homologando los datos en "clusters" o conjuntos homogéneos agrupados por materiales en similar sentido, se busca hacer un análisis del contenido de las entrevistas y del documento institucional para que sean de-construidos de sentido. Primeramente, se hace una interpretación que conlleva un re-construcción, pasando de una interpretación latente, a una interpretación manifiesta (Echevarría, 2005: 9).

El proceso de análisis de datos se sustentó en la codificación teórica que de acuerdo a Hernández (2014: 195-205) tiene como base las transcripciones de las entrevistas para después analizarlos y codificarlas. Para el caso de la cultura organizacional se utilizó una codificación selectiva basada en la teoría de Cameron y Quinn (2006) y el modelo hipotético-deductivo, ya que este considera que la investigación debe partir de un cuerpo teórico y de ahí, decidir qué hechos o datos hay que recopilar. En el caso de la inteligencia competitiva se utilizó una codificación abierta basados en el ciclo de García (2011) y posteriormente en una codificación selectiva creando categorías en función al control o flexibilidad del acopio de la información para fines institucionales. Al igual que en la codificación de la CO, para la IC se utilizó el modelo hipotético-deductivo por el cuerpo teórico con el cuál se recopiló y analizó la información.

Se presenta a continuación los resultados en cada una de las temáticas del objetivo de la investigación.

\subsection{Cultura organizacional}

De las entrevistas realizadas a docentes, directivos y administrativos, la codificación y análisis se sustentó en los tipos de CO de acuerdo a Cameron y Quinn (2006). En el cuadro 2 se incluyen las respuestas. La codificación de docentes se incluye como DO, la de administrativos como ADM y la de directivos como DIR.

\section{Cuadro 2. Dimensiones y tipos de CO de directivos, administrativos y docentes}

\begin{tabular}{|c|c|c|c|c|}
\hline & Clan & Adhocracia & Mercado & Jerárquica \\
\hline $\begin{array}{c}\text { Característica } \\
\text { dominante }\end{array}$ & DO es una familia & & $\begin{array}{l}\text { DO enfocada a in- } \\
\text { vestigación y CA } \\
\text { DIR valores externos }\end{array}$ & $\begin{array}{l}\text { DO, formación, do- } \\
\text { cencia, tradicional } \\
\text { DIR, compromiso } \\
\text { ADM compromiso, } \\
\text { tradicional }\end{array}$ \\
\hline Liderazgo & $\begin{array}{l}\text { DO proactivo según } \\
\text { necesidades } \\
\text { DIR cómodo, puer- } \\
\text { tas abiertas } \\
\text { ADM de unión y } \\
\text { coordinación }\end{array}$ & $\begin{array}{l}\text { DO situacional } \\
\text { DIR visionario }\end{array}$ & & $\begin{array}{c}\text { DO involuntario } \\
\text { ADM formal, institu- } \\
\text { cional } \\
\text { DIR institucional, } \\
\text { mandatos }\end{array}$ \\
\hline
\end{tabular}




\begin{tabular}{|c|c|c|c|c|}
\hline & Clan & Adhocracia & Mercado & Jerárquica \\
\hline $\begin{array}{l}\text { Administración de } \\
\text { empleados }\end{array}$ & $\begin{array}{l}\text { DO: equipo pos } \\
\text { simpatías, diálogo, } \\
\text { convenciendo } \\
\text { DIR tbjo equipo } \\
\text { ADM dialogo, em- } \\
\text { patía }\end{array}$ & $\begin{array}{l}\text { DO tbjo equipo se- } \\
\text { leccionando personal }\end{array}$ & & $\begin{array}{l}\text { DO se impone } \\
\text { ADM resistencia } \\
\text { DIR convenciendo en } \\
\text { base a resultados }\end{array}$ \\
\hline Cohesión & $\begin{array}{l}\text { DO en el lema y a } \\
\text { través de poderes } \\
\text { fácticos }\end{array}$ & & $\begin{array}{l}\text { DO trabajo CA } \\
\text { constante } \\
\text { ADM resistencia por } \\
\text { parte de maestros }\end{array}$ & $\begin{array}{l}\text { DO, DIR, ADM } \\
\text { normatividad y } \\
\text { procedimientos }\end{array}$ \\
\hline Énfasis estratégico & $\begin{array}{c}\text { DO: equipo a través } \\
\text { de tribus } \\
\text { DIR división del } \\
\text { trabajo }\end{array}$ & $\begin{array}{l}\text { ADM se detectan } \\
\text { áreas de oportuni- } \\
\text { dad } \\
\text { DIR si se aceptan } \\
\text { nuevas ideas }\end{array}$ & $\begin{array}{c}\text { ADM trabajo de CA } \\
\text { y maestros depende } \\
\text { de investigación }\end{array}$ & $\begin{array}{l}\text { DO, DIR, ADM tradi- } \\
\text { cional }\end{array}$ \\
\hline Criterios de éxito & $\begin{array}{l}\text { DO: intereses comu- } \\
\text { nes, individualistas } \\
\text { DIR buena comuni- } \\
\text { cación } \\
\text { ADM falta comuni- } \\
\text { cación }\end{array}$ & & $\begin{array}{c}\text { DO hacer redes, CA } \\
\text { y dirigir tesis } \\
\text { DIR trabajo en CA y } \\
\text { academias }\end{array}$ & $\begin{array}{c}\text { DO procedimientos } \\
\text { informales, claustro } \\
\text { DIR procedimientos } \\
\text { formales e infor- } \\
\text { males } \\
\text { ADM a través del } \\
\text { rector }\end{array}$ \\
\hline
\end{tabular}

Fuente: elaboración propia con base en entrevistas y categorización de acuerdo a Cameron y Quinn (2006)

Del análisis de los resultados de la muestra teórica se encontró lo siguiente:

Los docentes manifestaron una cultura jerárquica, de clan y mercado, de acuerdo a los informantes. Los valores se refieren a la docencia, formación y la tradición en el sentido jerárquico. Pero también expresaron valores de correspondiente a una cultura de mercado: la investigación y trabajo en los cuerpos académicos, que le dan cohesión de la manera en que se trabaja en la creación de redes e igualmente las tareas propias de los cuerpos académicos, dándole cohesión en la práctica constante. Expresaron que se les toma en cuenta, en la toma de decisiones, aunque señalan que se debe mejorar.

La cultura de clan, se expresa en los valores jerárquicos, pero hay una diferencia en la cohesión, para los docentes es una familia y en el lema de la institución, el trabajo de los grupos, donde se negocia con las tribus. El liderazgo es ejemplar, proactivo y de acuerdo a las necesidades del grupo. La cohesión se relaciona de la manera en que se estimula a las necesidades. Se negocia dialogando, informado de la toma de decisiones y se trabaja en equipo, a través de las tribus y uniendo simpatías. La manifestación del trabajo es interés común, pero a la vez individualista y siempre son los mismos.

De la cultura jerárquica distinguen con claridad que es formal con normatividad (varios mencionaron la ley orgánica) y relacionan con cartas descriptivas para dar clases. El liderazgo es involuntario y se impone, no se negocia. Los logros se reconocen personalmente y en ceremonias como el claustro. 
Para los administrativos, la cultura es transitoria pasando de la jerárquica, a la de clan y de mercado. Señalan como valores primordiales los siguientes: la tradición, servicio y compromiso. El liderazgo es formal, opaca a los demás, se negocia o no está claro el proceso, identifican resistencia al mismos, están formalizados los valores en las normas tale como: la ley orgánica y los planes institucionales. Los logros se dan a conocer por medios formales y el trabajo en equipo es de acuerdo a procesos definidos.

Pero manifiesta a la vez una cultura de clan, en el liderazgo y la forma en que se negocia con los diferentes grupos: manifestaron que es compartido entre los otros directivos y además de que se negocia reconociendo los logros y generando empatía, el trabajo en equipo señalan que hay una falta de comunicación.

También se evidencio un proceso de cambio hacia la cultura de mercado orientada hacia el exterior. En que la negociación se está buscado un proceso de cambio, y que la resistencia de los docentes hacia los planes institucionales, orientado a la investigación y el trabajo en cuerpos académicos, buscando propuestas de los participantes, aunque sea desde el claustro.

Los directivos expresaron valores referentes a la cultura de clan y jerárquica, así como de la cultura de mercado. Indicaron que el valor principal es el compromiso, servicio, trabajo y honestidad, se mencionó que también existen valores externos a la organización, que corresponde a una cultura de mercado.

En el liderazgo con los que se identificaron fueron: el ejemplar, que los subordinados estén cómodos. Pero expresaron que hay mandatos y un orden institucional. Si este tipo de valores y liderazgo favorece la cohesión, unos expresaron que sí, porque es a puertas abiertas, pero, señalaron que es ambiguo. La negociación es en base a los resultados, el trabajo en equipo está en los procesos, manifestándose en la cohesión, que se da la formalidad y la ley orgánica de la institución. El reconocimiento de los logros es formal e informal dependiendo de las circunstancias, se puede expresar en eventos e informes de los directivos. Todas estas categorías corresponden a una cultura jerárquica. En las entrevistas se manifestaron valores como ser visionario, y que le da cohesión de la manera que se aceptan ideas nuevas. Correspondiendo a una cultura adhocrática.

En el cuadro 3 se presenta el análisis de la CO en el Plan de Desarrollo Universitario 2011-2021 (UACH, 2011) de acuerdo a los tipos de CO de Cameron y Quinn (2006) se analizaron en dos partes: al 2011 cuando presentan un diagnóstico de esa fecha y al 2021, fecha en que concluye el plan.

\section{Cuadro 3. Tipos de CO en el Plan de Desarrollo Universitario 2011-2021}

\begin{tabular}{|c|c|c|}
\hline & IES 2011 & IES 2021 \\
\hline Clan & $\begin{array}{l}\text { valores: identidad universitaria, solidaridad y } \\
\text { formación integral } \\
\text { liderazgo: ética, honestidad, verdad, tolerancia } \\
\text { estrategia coherente entre discurso y acción } \\
\text { trabajo en equipo colegiado }\end{array}$ & $\begin{array}{l}\text { valores: identidad universitaria, RSU, medio } \\
\qquad \text { ambiente } \\
\text { estrategia: universidad saludable e incluyente }\end{array}$ \\
\hline Jerárquica & $\begin{array}{l}\text { estrategias en evaluación } \\
\text { trabajo en equipo con eficiencia en procesos } \\
\text { académicos }\end{array}$ & $\begin{array}{l}\text { trabajo en equipo con esquemas y procedi- } \\
\text { mientos vigentes de evaluación y acreditación }\end{array}$ \\
\hline
\end{tabular}




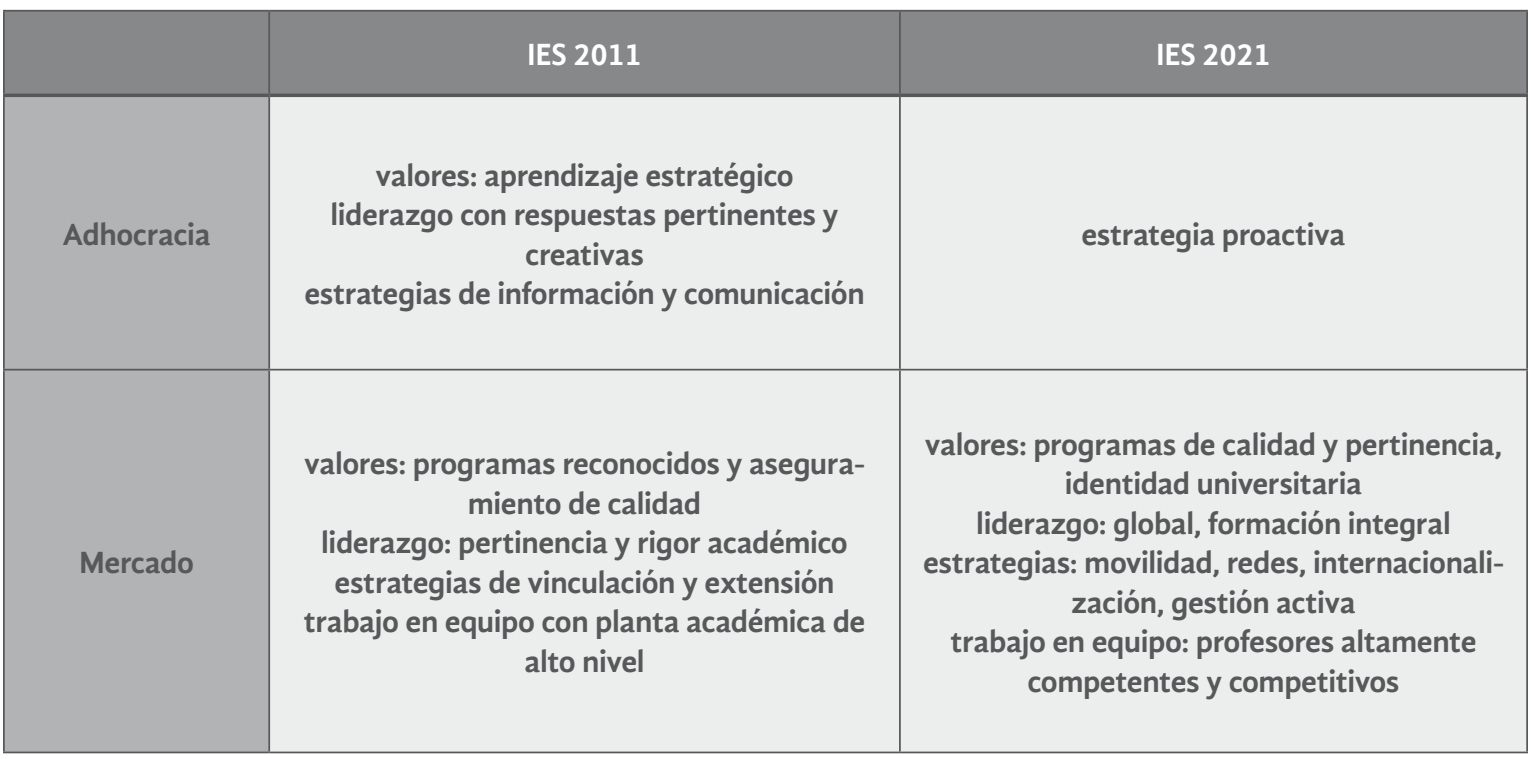

Fuente: elaboración propia del análisis de documento institucional y de acuerdo a Cameron y Quinn (2006)

El análisis de este documento institucional de acuerdo a la categorización de Cameron y Quinn (2006) revela que en su diagnóstico 2011 lo siguiente:

1. Cultura de clan: se identifican por valores como la identidad y solidaridad con la institución, el liderazgo es dirigido a valores éticos, honestidad, tolerancia y coherente con el discurso y las acciones y el trabajo es colegiado.

2. Cultura jerárquica: se identifica en los procesos del trabajo en equipo y evaluación de los mismos.

3. Cultura adhocrática: se identifica en los valores de estrategia, liderazgo que busca la creatividad y con una búsqueda de la información.

4. Cultura de mercado: generalmente enfocado en la calidad, ya sea de sus profesores, que se logra en ejercicio de liderazgo basados es la pertinencia y rigor académico con estrategias de vinculación y extensión, con un equipo de trabajo de una planta académica de alto nivel.

En cuanto a la proyección 2021, el análisis nos presenta que se identifican tres culturas: la cultura de clan está fundamentada en los valores de una identidad y en la promoción de estrategias relacionadas con valores sociales de la organización, que buscan fortalecer esa identidad. La cultura de jerárquica en cuanto la evaluación y aseguramiento de proceso. Pero mayormente se visualiza como con cultura de mercado, en base a la calidad de sus programas y una planta docente altamente competente y competitiva. Sus estrategias son: la movilidad e internacionalización, gestión activa de todos sus miembros. El liderazgo se ejerce: con perspectiva global, la formación integral de profesionistas, científicos y líderes. 


\subsection{Inteligencia competitiva}

Para el análisis de la inteligencia competitiva se crearon categorías de acuerdo al ciclo de la IC de García (2011) en función al control o flexibilidad del acopio de la información para fines institucionales. La numeración es de mayor a menos, correspondiendo al número mayor el mayor control para el acopio de la información, creando las siguientes categorías:

5. Institucionalizada y normada. Las normas y procedimientos están institucionalizados ya sea en la ley orgánica de la misma, en los reglamentos y el plan de desarrollo institucional

4. Institucionalizada y no normada. Se encuentra en las pautas de desarrollo de la misma, como misión, por ejemplo: el incremento de matrícula, el trabajo en los modelos educativos.

3. La institución lo menciona, pero se trabaja de manera individual. No se encuentra dentro de las normas de un cuadro normativo, sin embargo, los miembros de la misma consideran que forma parte de la misión misma de la organización.

2. No se trabaja institucionalmente sino de manera individual. No es parte de ninguna manera normada, pero los miembros lo buscan activamente en reacción a los diferentes procesos que la misma desarrolla.

1. No se realiza en la institución o se desconoce. No es parte de la estructura formal de la institución, pero se desarrolla de manera individual. Pero también existe un grupo de normas o procesos que se desconoce de ellos.

Los resultados se agrupan de acuerdo a la información que les requiere COPAES, CIEES y PNPC siendo esta en relación a la publicación científica de sus académicos, los procesos de acreditación y evaluación a los que se ven sometidos, la obtención de recurso e infraestructura y la colaboración e internacionalización relacionada con la vinculación de los académicos. El análisis en cuanto a donde y quienes recopilan la información, se encontraron los siguientes resultados de acuerdo a la categorización elaborada.

\section{5.-Institucionalizada y normada.}

Sobre la publicación científica reconocen algunos que las publicaciones se encuentran en investigación y posgrado, que se encuentra en el perfil de cada académico investigador importantes para el proceso de acreditación de la organización.

Que se almacenan en las bibliotecas en las editoriales y en planeación y desarrollo de la institución. Que la interpretación no se hace, que hay una falta de articulación.

Sobre acreditación y evaluación, la información relevante para los procesos, la determina planeación y desarrollo y secretaria académica. Señalan que el almacenamiento de encuentra en las facultades y las direcciones de investigación y posgrado y planeación y que de ahí se hace el análisis de la información, pero que no existe una estrategia integral

Sobre los recursos e infraestructura y la forma en que se obtienen, señalan que, si hay una divulgación, que se almacena la información de la obtención de dichos recursos en la dirección académica, y que el nivel jerárquico de quien recibe dicha información es variado, desde el rector y directores de diferentes áreas y departamentos. 
Sobre la colaboración e internacionalización, se señala que la detención de la información consiste primordialmente en: información, financiar a cuerpos académicos, así como formalizar la colaboración, por medio de contratos. Que en servicios internacionales se almacena y está en una plataforma y que es ahí donde se interpreta y analiza la información.

\section{4.- Institucionalizada y no normada.}

Que la publicación científica, depende de cada académico, que cuando está el proceso de acreditación se realiza aparentemente la recopilación de la información. La interpretación se realiza estadísticamente en investigación y posgrado.

Sobre la acreditación y evaluación, señalan que se crean gestores académicos, que en algunas facultades y en direcciones se almacena la información y el análisis e interpretación, se da en todas esas instancias.

Sobre la obtención de recursos e infraestructura se menciona que se está desarrollando una plataforma o sistema SAFE para incorporar la información. Que el análisis lo hacen en cada departamento.

Sobre la colaboración e internacionalización, que cada facultad tiene su información y que la interpretación corresponde a cada unidad, Pero la interpretación les corresponde a los secretarios de cada unidad académica y los directores de la misma.

\section{3.- La institución lo menciona, pero se trabaja a nivel individual.}

Sobre la publicación científica, se detecta la información en el perfil PRODEP de cada maestro y que le corresponde al área de planeación de la institución. Que se almacena en las publicaciones de las revistas de la institución. En cuanto a la interpretación, nadie de los entrevistados ofreció una respuesta.

Sobre los recursos infraestructura, depende de cada cuerpo académico y se almacena en su perfil PRODEP, así como colaboran y se internacionalizan

\section{2.- No se trabaja a nivel institucional, sino de manera individual.}

En la producción científica se encontró: que la creación de redes de investigación es de manera individual a cada docente o investigador. Los recursos e infraestructura algunos dependen de su trabajo EN cuerpos académicos y sus redes. El almacenamiento está en la información de cada cuerpo académico.

\section{1.- No se realiza en la institución o se desconoce.}

En la producción científica, mencionan que la información que se obtenga depende de las áreas de conocimientos, o que cada maestro trabaja de manera individual. En las acreditación y evaluación, se trabaja en la manera de conocer la información. Saben que existe información sobre los recursos y la infraestructura, así como de colaboración e internacionalización, pero algunos lo desconocen. Algunos de los informantes desconocen, además, donde se almacena la información en las siguientes áreas: recursos e infraestructura, la colaboración e internacionalización. Además, si se hace un análisis y nivel jerárquico a donde se lleva la información.

El análisis en cuanto a estrategias y mecanismos de respuesta en las diferentes etapas del ciclo de IC, se encontraron los siguientes resultados de acuerdo a la categorización elaborada.

\section{5.- Institucionalizada y normada.}

Las estrategias para incentivar la publicación científica están ligadas a las becas al desempeño. La estrategia en sí, es el área de oportunidad. Sobre la organización y almacenamiento, señalan algunos, que no

existe un mecanismo o estrategia para incentivarse, también se señala que se está buscando implementar un repositorio digital. Que la interpretación y análisis, deben hacerlo los funcionarios. 
Sobre la acreditación y evaluación: señalan que la detención de la información está en los indicadores de las acreditadoras. Que la organización y almacenamiento, se comparten los archivos de cada unidad académica, pero algunos señalan que es insuficiente. Y la interpretación y análisis, la estrategia se refiere a cursos cuando se da el proceso de acreditación.

Recursos e infraestructura: la estrategia de la detección de la información es necesaria para los presupuestos de las facultades y cada vez que se actualiza los currículos. El almacenamiento está a disposición cada vez que lo solicitan. En colaboración e internacionalización el almacenamiento está en las acreditadoras.

\section{4.- Institucionalizado y no normado.}

La estrategia para detectar la información de publicación científica es en las editoriales además de los incentivos de la beca. El análisis de la información y nivel jerárquico y quien le entrega los informes señalan que se les da todos.

Sobre la acreditación y evaluación la detención de la relevancia de la información está en los lineamientos de las acreditadoras y la estrategia sobre el almacenamiento está en las secretarias de planeación e investigación y se comparte a los cuerpos académicos.

\section{3.- La institución lo menciona, pero se trabaja nivel individual.}

La estrategia de la recopilación de la información, se da cada vez que piden apoyo para la publicación y cada facultad lo incentiva. Sobre la acreditación y evaluación señalan que depende del interés del maestro.

\section{2.- No se trabaja a nivel institucional, sino individual.}

Sobre las publicaciones y la creación de redes depende de cada maestro al igual sobre la obtención de los recursos e infraestructura. Sobre la acreditación y evaluación se almacena sobre los logros de los cuerpos académicos y se les comparte y también, que cada facultad debe hacer el análisis.

1.- No se realiza en la institución o se desconoce.

Sobre las publicaciones e investigación, desconocen si existe alguna estrategia. Que no se les apoya en el proceso de publicación. Desconocen si existe estrategias sobre la acreditación y evaluación. Y sobre los recursos e infraestructura, las estrategias son espontaneas.

Sobre las estrategias del almacenamiento, es cada vez que hay un registro de las investigaciones o cuando buscas el perfil del maestro. En la acreditación y evaluación, no se les informa. Desconocen de alguna estrategia del almacenamiento de recursos e infraestructura, así como de la colaboración e internalización. Sobre el análisis y nivel jerárquico, no existe ninguna y el maestro es quien interpreta y sobre la acreditación y evaluación, no lo saben.

En el cuadro 4 se presenta el análisis de las estrategias de gestión del Plan de Desarrollo Universitario 2011-2021 (UACH, 2011) divididas en dos partes: al 2011 cuando presentan un diagnóstico de esa fecha $y$ al 2021, fecha en que concluye el plan.

\section{Cuadro 4. Estrategias de gestión del Plan de Desarrollo Universitario 2011-2021}

\begin{tabular}{|c|c|}
\hline Gestión en 2011 & Gestión 2021 \\
\hline $\begin{array}{l}\text { 1.- No se cuenta con sistema consolidado de gestión para } \\
\text { la mejora continua y aseguramiento de la calidad de las } \\
\text { funciones administrativas. }\end{array}$ & $\begin{array}{l}\text { 1.- Orientación hacia el mismo plan, creación de } \\
\text { procesos adaptativos, trabajo colegiado, esquemas de } \\
\text { seguimiento. }\end{array}$ \\
\hline
\end{tabular}




\begin{tabular}{|c|c|}
\hline Gestión en 2011 & Gestión 2021 \\
\hline 2.-El porcentaje de posgrados de calidad es bajo. & $\begin{array}{l}\text { 2.- Con sistema de información y de indicadores de } \\
\text { desempeño, marco normativo actualizado, procesos } \\
\text { de certificación en normas internacionales. }\end{array}$ \\
\hline $\begin{array}{l}\text { 3.- Las políticas institucionales para impulsar la supera- } \\
\text { ción continua es insuficiente. }\end{array}$ & $\begin{array}{l}\text { 3.-Desarrollo de un sistema integral de información } \\
\text { eficiente y confiable que sustente la toma de decisio- } \\
\text { nes. }\end{array}$ \\
\hline $\begin{array}{l}\text { 4.- El trabajo colegiado es insuficiente para promover la } \\
\text { mejora continua. }\end{array}$ & 4.-Fortalecer el programa de estímulos. \\
\hline 5.- Escasa colaboración y movilidad académica. & $\begin{array}{l}\text { 5.- Implementar procedimientos sistematizados y } \\
\text { permanentemente actualizados. }\end{array}$ \\
\hline $\begin{array}{l}\text { 6.- La infraestructura y equipamiento de la universidad } \\
\text { no es suficiente. }\end{array}$ & 6.- Desarrollar sistemas de comunicación efectivos. \\
\hline $\begin{array}{l}\text { 7.- La colaboración de la universidad con instituciones } \\
\text { nacionales y extranjeras es incipiente. }\end{array}$ & $\begin{array}{l}\text { 7.- Identificar elementos necesarios para la incorpora- } \\
\text { ción de la institución en rankings de calidad. }\end{array}$ \\
\hline $\begin{array}{l}\text { 8.- Los esquemas y programas vigentes de vinculación } \\
\text { son insuficientes. }\end{array}$ & 8.- Fortalecer un sistema de evaluación interna \\
\hline \multirow[t]{3}{*}{$\begin{array}{l}\text { 9.- La capacidad de la universidad para generación y apli- } \\
\text { cación es insuficiente. } 30 \% \text { de profesores con doctorado, } \\
\text { solo } 8 \% \text { en SNI y } 5 \% \text { de CAs consolidados. }\end{array}$} & $\begin{array}{l}\text { 9.- Fortalecer la construcción de sistemas de infor- } \\
\text { mación administrativa y favorecer el uso de bases de } \\
\text { datos. }\end{array}$ \\
\hline & $\begin{array}{l}\text { 10.- Diseñar e implementar instrumentos para eva- } \\
\text { luar el desempeño del personal académico. }\end{array}$ \\
\hline & $\begin{array}{l}\text { 11.-Gestion de recursos para el desarrollo institu- } \\
\text { cional y el adecuado cumplimiento de las funciones } \\
\text { institucionales. }\end{array}$ \\
\hline
\end{tabular}

Fuente: elaboración propia con base en Plan de Desarrollo Universitario 2011-2021 (UACH, 2011)

Desde un enfoque de IC, las estrategias de gestión en el documento institucional analizado, tienden en el 2021 a ser institucionalizadas y normadas.

\section{Discusión y Conclusión}

En el presente estudio se realizó una identificación y descripción de las culturas dominantes de una IES en el norte de México, considerando que este diagnóstico de acuerdo a Cameron y Quinn (2006: 83) es de vital importancia para un proceso de cambio o como en el caso de esta IES, que ha sido afectada por cambios externos como la evaluación y acreditación de programas, así como por la calidad universitaria y específicamente para este estudio, como se dan sus procesos de IC, analizando para este fin su documento 
institucional denominado Plan de Desarrollo Universitario 2011-2021 (UACH, 2011) y mediante entrevistas semiestructuradas.

En relación a la cultura organizacional de esta IES, el documento institucional en su proyección 2021 se identifican tres culturas: cultura de clan, cultura jerárquica y de manera dominante la cultura de mercado, que está orientado a fuerzas externas de calidad, como su principal objetivo. Cameron y Quinn mencionan que una organización con un ambiente identificada como Clan y una cultura débil de mercado encontrara que es difícil la adaptación a un ambiente cada vez más competitivo y es necesario desarrollar estrategias de cambio para enfrentar las nuevas demandas de cambio (Cameron y Quinn, 2006: 71), en ese sentido la proyección 2021 de esta IES que presenta de manera dominante la cultura de mercado, lo que favorecerá que siga desarrollándose en al ambiente competitivo de las IES.

Este enfoque institucional al 2021 contrasta con la forma en que los entrevistados identifican la CO en 2017:

a.- Los directivos expresan valores, estrategias, liderazgo y metas de una organización jerárquica: se consideran buenos administradores y buscan mantener una organización que se centra en mantener una organización eficiente con metas a largo plazo centradas en estabilidad y resultados, formalizado con valores de estructuras y procedimientos de control.

b.- Los administradores evidencian un tránsito de diferentes culturas, pero se puede ver que manifiestan la cultura de mercado: los valores están orientados a resultados, buscan lograr el trabajo, orientados a metas competitivas. El liderazgo es producir resultados y buscan las metas a largo plazo diseñadas en el plan institucional.

c.- Los docentes se centran en dos culturas: la jerárquica y la de clan, con una preponderancia hacia la última ya que la consideran un lugar amigable como de familia, el liderazgo es de mentor, proveedores, y es importante la tradición, en vistas de desarrollar recursos humanos y ser sensibles con el servicio.

Este contraste en la identificación de la $\mathrm{CO}$ a través de su documento estratégico y las entrevistas de los participantes, revela que mientras la proyección 2021 si identifica las culturas de clan, jerárquica y de manera dominante la de mercado, en 2017 a cuatro años de la fecha de la proyección, la CO que ven docentes, administradores y directivos es preponderantemente jerárquica, aunque si vislumbran la cultura de mercado como algo que está sucediendo, sin ubicar con claridad estrategias institucionales que les permita transitar a ese cambio. Por lo que se concluye que:

1.- La cultura declarada en el análisis del plan institucional no corresponde con la que han declarado los entrevistados. En el plan se visualizan como una organización altamente competitiva y los entrevistados solo un grupo manifestó ese tipo de organización.

2.-La organización manifestó un plan que busca un cambio para el 2021, pero solo un grupo (administrativos), manifestaron conocerlo. Los demás no lo mencionaron en forma consensada, además de que desconocen acciones para la implementación de dichos cambios, generalmente hablaron de incentivos en relación a becas u obtención de beneficios.

Este hallazgo es importante, ya que a raíz de la identificación y diagnóstico de las culturas de una organización es que se está en mejores condiciones para definir las estrategias necesarias para llevar a cabo la transición necesaria para el cambio de cultura que se busca e involucrar a los miembros para que comprendan las ventajas de hacerlo. 
Otro hallazgo importante en relación a la identificación de la CO actual en la IES de estudio, es la no inclusión de la cultura adhocrática. Los estudios de Cameron y Quinn, señalan que el desarrollar o no desarrollar una cultura adhocratica es un problema en la mayoría de las organizaciones. Generalmente se gravitan entorno a las jerárquicas o de mercado, una vez que se encuentran dominadas por ese tipo parece que es difícil lograr una cultura flexible representada por la de clan y adhocratica que se caracterizan por esos valores. Los efectos sobre la dirección de la misma es que hay una desconexión entre el liderazgo y la gestión dentro de las mismas (Cameron y Quinn, 2006: 80).

Los líderes que no son gestores o viceversa los gestores que no ejercen un liderazgo tienden a un fracaso puesto que un cambio necesita de ambos. Particularmente señalan que la efectividad de una IES se comportaba en alto grado en aquellas en donde existía un énfasis en la innovación (adhocracia) y cambio. Pero a la vez tenían estabilidad y control (jerárquica). Además, este tipo de organizaciones desarrollaban una forma efectiva de soporte en el desarrollo de sus miembros (clan). Pero existía una demanda de resultados (mercado), con una estrategia del logro de los mismos (Cameron y Quinn, 2006: 81)

En relación con la inteligencia competitiva de esta IES, el documento institucional en su diagnóstico 2011 mencionaron como debilidades que no se cuenta con un sistema consolidado de gestión para la mejora continua y aseguramiento de la calidad de las funciones administrativas y una insuficiente capacidad de generación y aplicación del conocimiento, mientras que su proyección al 2021 las estrategias de gestión serán institucionalizadas y normadas.

En el análisis de las entrevistas, se expresó que las estrategias habían tenido un comportamiento adaptativo, así como en la indigencia en general y manifestaron que los resultados habían sido positivos por el incremento en todos los rubros: 1.- las publicaciones, 2.- evaluación y acreditación, 3.- gestión, 4.-la movilidad e internacionalización. Pero también revelaron que existe una inconsistencia en la medida que los análisis de la información y los niveles de jerárquico a los que llegan, desconocen si se realiza, aunque suponen que sí, pero hay que señalar que los administrativos expresaron que si se hacia los análisis y que la información se hacía llegar a todos los niveles o si eran solicitadas. Es en los docentes donde se apreció, que existe un desconocimiento de todas las etapas del ciclo de IC. De manera general se encontró falta de conocimiento sobre la IC que realiza la institución y unificación de criterios sobre qué departamento la recopila. Aunque la institución ha declarado en su plan institucional como una necesidad la información que requiere, existe un desconocimiento entre lo planificado y el conocimiento de la estrategia de recogida de la información.

Esto puede ser consistente con el tipo de cultura dominante en la institución, ya que como lo señalan Cameron y Quinn (2006: 84-105) los procesos de cambio se inician con la identificación real de las culturas dominantes, que por un lado en su plan institucional manifiesta en 2011 una cultura dominante jerárquica y buscan ser una cultura de mercado en 2021. Por lo que, para poder lograr ese cambio, el apoyo de la IC en las diferentes etapas de su ciclo requiere que las estrategias estén claramente definidas, así como las áreas o departamentos que colecten la información y que esto, sea difundido y comunicado a todos los integrantes de la institución. Para los teóricos una cultura que sea más efectiva en la adaptación de la IC, y producir un cambio dentro de la misma es la que considere como una estrategia definida por los objetivos que la organización considere como importantes. Las tácticas que se deben considerar para un cambio en la organización son: 1.- una estrategia, por parte de los directivos, 2.- la estructura, relacionada a la organización misma, si es centralizada con una jerarquía vertical o descentralizada con una jerarquía 
horizontal, 3.- habilidades, relacionada a la capacidad del personal y la administración, 4.- El personal, los recursos humanos que necesitan ser capacitados, 5.- sistematización, los procesos y procedimientos, herramientas y comunicación necesarios para conducir la organización, 6.- El estilo, promoción del uso de la IC, en los que toman las decisiones y 6.- Valores compartidos, relacionado a la cultura de la organización, ya que es un requisito en entender la necesidad de IC (La Palme, 2003: 2015-2017). En un estudio de Bogdanowicz (2014: 65) de la transformación de organizaciones de un liderazgo efectivo, se buscó que se identificara el grado de flexibilidad de las mismas para buscar favorecer el uso de una IC, ya que las culturas de clan y adocrática buscan la innovación y la competitividad, además de que favorece la adaptabilidad de la misma.

La efectividad de la implementación de una IC (Kbrom, 2004: 64) está relacionado con la CO en la manera en que se determina la necesidad de utilizar la información relativa para obtener una ventaja competitiva. Una organización con una cultura débil, es aquella que no encuentra una relación entre la estrategia y las culturas dominantes y crea un ambiente que no favorece el uso de información ya que no valora la importancia de la misma. De acuerdo a Kbrom (2004: 64-67) en un estudio por instituciones gubernamentales se encontró que el uso deficiente de la información proporcionada se relacionaba a la falta de concientización de la utilizad de la misma.

Siguiendo la clasificación de organizaciones de IC en positivas y negativas, podemos encontrar que tipo de $\mathrm{CO}$ mejor valora la utilización de una estrategia de información. Una cultura positiva esta centrada en una aproximación de los que hacen un análisis de la información con los encargados de procesar y analizar la información. Las negativas son aquellas que no han generado el valor cultural del proceso estratégico de la información (Moffact y Fleisher, 2003: 278)

Finalmente, y de acuerdo con los resultados y conclusiones obtenidos, lograr este cambio cultural se puede reducir en seis pasos importantes de acuerdo a Cameron y Quinn (2006: 84-105): 1.-llegar al consenso de qué tipo de cultura existe, 2.-llegar al consenso del futuro de la organización, 3.- determinar lo que no significa, así como lo que será el futuro cultural de la organización, 4.- Identificar historias ilustrativas, de organizaciones que lograron hacer los cambios. 5.- Desarrollar un plan de acción y 6.- Desarrollar una implementación de un plan. Una historia ilustrativa es el estudio de cambio cultural e inteligencia competitiva en empresas de Latvia (Cekuls, 2015: 244-256), basadas en los tipos de cultura de Cameron y Quinn (2006) donde se encontró que el liderazgo es importante ya que son los lideres los que implementas valores y es preferible que quienes ejercen liderazgo vigilen los nuevos valores de la organización, con base en comunicación abierta, colaboración y confianza. En ese sentido, este estudio aporta la identificación de las CO dominantes de una IES en el norte de México y el estado actual de la IC contrastada con su diagnóstico en 2011 y su proyección al 2021 con la que pretenden fortalecer y consolidar su calidad universitaria y permite a partir de esta identificación, clarificar el trabajo necesario a realizar para lograr su proyección al 2021.

Nuevos estudios que relacionen CO e IC en IES que han logrado transitar de un tipo de cultura a otra de mercado son necesarios, ya que la mayoría de los estudios a la fecha son en organizaciones industriales o comerciales. Se sugiere también, continuar esta investigación de forma cuantitativa para poder describir asociaciones entre los constructos y clasificaciones desde un encuadre mixto. Lo que permitirá ampliar la clasificación de las culturas dentro de la IES objeto de estudio, para poder diagnosticar eficientemente las culturas dominantes, con datos cuantificables y bajo modelos de confiabilidad estadística. De esa manera las estrategias tomadas en el plan institucional tendrían un mayor alcance, se podría generar 
una clasificación de la utilización de la IC. Por último, es importante realizar en un futuro, la divulgación del seguimiento de los planes de cambio de la IES presentada en esta investigación, ya que sus esfuerzos y trabajo permitirán a otras IES en condiciones y enfoque similar, verla como una historia ilustrativa de organizaciones que lograron hacer esos cambios.

\section{Referencias}

Alvesson, Mats. 2002. Understanding organizational culture. Londres: SAGE Publications. Best, John. 1970. Como investigar en educación. Madrid: Ediciones Morata.

Bikmoradi, Alii, Mats Brommels, Alireza Shoghli, Davoud Khorasani Zavareh y Italo Masiello. 2009. Organizational culture, values, and routines in Iranian medical school. Higher education, 57 (4): 417-427. DOI: http://doi.org/10.1007/s10734-008-9152-2

Blaxter, Loraine, Christina Hughes y Malcolm Tight. 2000. Como se hace una investigación. Barcelona: Gedisa.

Bogdanowicz, Maryla. 2004. Organizational culture as a source of competitive advantage - case study of a telecommunication company in Poland. International Journal of Contemporary Management, 13 (3): 53-66.

Bryman, Alan y Emma Bell. 2015. Business research methods. Oxford: Oxford University Press.

Cáceres, Pablo. 2003. Análisis cualitativo de contenido: una alternativa metodológica alcanzable. Psicoperspectivas, 2: 53-82.

Calof, Jonathan. 1999. Overcoming competitive inteligencie barriers: A SCIP tool kit. Competitive Inteligence Review, 10 (1): 71-78.

Cameron, Kim y Robert Quinn. 2006. Diagnosing and changing organizational culture. San francisco, CA: Jossey-Bass.

Cekuls, Andrejs. 2015. Leadership values in transformation of organizational culture to implement competitive intelligence management: the trust building through organizational culture. European Integration Studies, (9): 244-257. DOI: http://doi.org/10.5755/j01.eis.0.9.12811

Chebbi, Jihene y Zeined Ammar. 2015. Influence of organizational culture on competitive intelligence practice: a conceptual framework. International Journal of Innovation, Management and Technology, 6 (1): 35-40. DOI: http://doi.org/10.7763/IJIMT.2015.V6.570

CIEES. 2018. Metodología CIEES. https://www.ciees.edu.mx/?catid=0\&id=24 (15 de febrero, 2018).

CONACYT. 2017. Normatividad. https://www.conacyt.gob.mx/index.php/el-conacyt/normatividad (15 de febrero, 2018).

COPAES. 2017. COPAES. https://www.copaes.org/identidad.php (15 de febrero, 2018).

Cory, Henneth. 1996. Can competitive intelligence lead to a sustainable competitive advantage? Competitive Intelligence Review, 7 (3): 45-55. DOI: http://doi.org/10.1002/cir.3880070309

Creswell, John. 1998. Qualitative inquiry and research design. choosing among five traditions. Thousand Oaks, Calfornia: SAGE Publications.

de la Cuesta, Carmen. 2006. La teoría fundamentada como herramienta de análisis. Cultura de los ciudadanos, 10 (20): 136-140. DOI: http://doi.org/10.14198/cuid.2006.20.19 
Denison, Daniel y Gretchen Spreitzer. 1991. Organizational culture and organizational development: a competing values approach. Research in Organizational Change and Development. 5:1-25

Dill, David. 2012. The management of academic culture revisited: integrating universities in an entrepreneurial age. En Managing reform in universities. Issues in higher education, editado por Stensaker B., Välimaa J., Sarrico C. Londres: Palgrave Macmillan, 222-237.

Echeverría, Genoveva. 2005. Apuntes docentes de metodología de investigación análisis. Universidad Academia de Humanismo Cristiano. Escuela de Psicología. Chile: Universidad Academia de Humanismo Cristiano.

García, Montserrat. 2011. Contribución de la inteligencia competitiva en el proceso de adaptación al EEES: el caso de las universidades españolas. Tesis doctoral, Barcelona: Universitat Oberta de Catalunya.

Giustozzi, Emilie y Betsy Van der Veer Martens. 2011. The new competitive intelligence agents: "Programming" competitive intelligence ethics into corporate cultures. Webology, 8 (2): 1-12.

Hatch, Mary y Majken Schultz. 1997. Relations between organizational culture, identity and image. European Journal of Marketing, 31 (5/6): 356-365. DOI: http://doi.org/10.1108/eb060636

Hernández, Rafael. 2014. La investigación cualitativa a través de entrevistas: su análisis mediante la teoría fundamentada. Cuestiones Pedagógicas, 23: 187-210.

Hernández, Roberto, Carlos Fernández y Pilar Baptista. 2014. Metodología de la investigación. Ciudad de México: McGraw-Hill.

Kbrom, Berhane. 2004. The relationship between organizational culture and competitive intelligence performance in the context of Eritrean trade and manufacturing industries. Tesis de Maestría, Stellenbosch: University of Stellenbosch.

La Palme, Kathleen. 2003. How can competitive intelligence best adapt to organizational chenge? En Controversies in competitive intelligence. The enduring issues, editado por Craig Fleisher y David Blenkhorn. Westport: Praeger, 1-33.

Martín-Crespo, Cristina y Belén Salamanca. 2007. El muestreo en la investigación cualitativa. NURE investigación, (27): 1-4.

Martínez, Miguel. 2018. Validez y confiabilidad en la metodología cualitativa. Paradigma, 27 (2): 1-13. Mejía, Julio. 2000. El muestreo en la investigación cualitativa. Investigaciones sociales, 5 (4): 165-180. Moffat, Lisa y Craig Fleisher. 2003. How can an organization's culture be changed to better support competitive intelience? En Controversies in competitive intelligence: the enduring issue, editado por Craig Fleisher y David Blenkhom. Westport: Praeger, 266-280.

Petrişor, Ioan y Natalia Ana Străin. 2012. Approaches on the competitive intelligence. The USV Annals of Economics and Public Administration, 13(1): 1-109.

Prior, Vernon. 2018. Glossary of terms used in competitive intelligence and knowledge management. Strategic and competitive intelligence professionals web page. http://c.ymcdn.com/sites/www.scip.org/resource/resmgr/White_Papers/Prior_Intelligence_Glossary_.pdf?hhSearchTerms=\%22definition+and+ competitive+and+intelligence\%22 (17 de febrero, 2018).

Schein, Edgar. 2004. Organizational culture and leadership. San Francisco: Josey Bass.

Simon, Neil. 1999. The effects of organizational culture on the IC process. Competitive Intelligence Review, 10 (1): 62-70. DOI: http://doi.org/10.1002/(SICI)1520-6386(199931)10:1<62::AID-CIR9>3.0.CO;2-B

Soto, Stephen. 2001. Competitive intelligence methods for systems and cultural analysis. Competitive 
- Revista de Ciencias Sociales y Humanidades. ISSN-P: 0188-9834 ISSN-E: 2395-8669.

Intelligence Review, 12 (3): 31-34. DOI: http://doi.org/10.1002/cir.1023

Tamayo y Tamayo, Mario. 2003. El proceso de la investigación científica. Ciudad de México: Limusa.

UACH. 2011. Plan de desarrollo universitario 2011-2021. http://www.uach.mx/planeacion/documentos/2011/08/15/pdu_2011-2021/ (13 de febrero de 2018).

UACH. 2018a. Historia. http://www.uach.mx/institucional_y_juridica/organizacion/2008/03/07/historia/ (18 de febrero de 2018).

UACH. 2018b. Estadística básica 2016. http://www.uach.mx/planeacion/2018/01/15/estadistica-basica-2016.pdf (18 de febrero de 2018).

Vasilachis, Irene. 2006. Estrategias de investigación cualitativa. Barcelona: Gedisa.

Wang, Camilla y Luis Borges. 2013. Does competitive intelligence matter? an anthropological way of thinking. International Journal of Business Anthropology, 4 (2): 76-84.

Weick, Karl. 1995. Sensemaking in organizations. Thousand Oaks: SAGE Publications. 